\title{
Décadrages Décadrages
}

cinéma, à travers champs Cinéma, à travers champs

$20 \mid 2012$

Peter Watkins

\section{Prosopopées - visages et paroles dans quelques films de Peter Watkins}

Mathias Lavin

\section{OpenEdition}

1 Journals

Édition électronique

URL : https://journals.openedition.org/decadrages/227

DOI : $10.4000 /$ decadrages. 227

ISSN : 2297-5977

Éditeur

Association Décadrages

Édition imprimée

Date de publication : 10 avril 2012

Pagination : 34-44

ISBN : 978-2-9700668-4-2

ISSN : 2235-7823

Référence électronique

Mathias Lavin, «Prosopopées - visages et paroles dans quelques films de Peter Watkins »,

Décadrages [En ligne], 20 | 2012, mis en ligne le 10 avril 2013, consulté le 03 avril 2022. URL : http://

journals.openedition.org/decadrages/227 ; DOI : https://doi.org/10.4000/decadrages.227

(B) Décadrages 


\section{Prosopopées - visages et paroles dans quelques films de Peter Watkins}

par Mathias Lavin

1 Edvard Munch, Ecrits, Dijon, Presses du réel, 2011, p. 104 ["L'arbre de la connaissance en bien et en mal. Carnet de croquis ", circa 19301935].

2 Voir Antoine de Baecque, "Peter Watkins en direct de I'histoire", L'histoire-caméra, Paris, Gallimard, 2008, pp. 207-261.
Je vois tous les êtres humains derrière leurs masques

Les visages calmes et souriants de pâles cadavres qui affairés se hâtent sur une route tortueuse dont la fin est la tombe (Edvard Munch) 1

Evocation empathique de l'insurrection hongroise de l'automne 1956, Forgotten Faces (1960), un des premiers courts métrages de Peter Watkins, offre une voie d'entrée féconde dans son œuvre. Réalisé près de Canterbury avec la troupe de théâtre amateur du Playcraft Group, le film adopte le style des actualités filmées en proposant une première esquisse de ses futurs "documentaires fictifs", selon l'expression d'Antoine de Baecque $^{2}$. Il possède en outre un titre significatif qui, de manière rétrospective, prend valeur de programme. En multipliant les figures singularisées par des gros plans, il interroge en effet ce qu'est un visage au cinéma, tout en indiquant une dimension collective induite par le pluriel. Au-delà du film "hongrois», cette question concerne une partie du travail du réalisateur qui présente de manière récurrente un resserrement du cadre sur la seule face humaine. A l'instar d'Edvard Munch qui souffrait d'agoraphobie, on a ainsi souvent l'impression que les corps filmés par Watkins ne supportent pas d'être inscrits dans un espace trop vaste, comme s'ils ne pouvaient exister qu'isolés par un gros plan. Ou alors ils sont insérés dans un cadre saturé les vouant à la fragmentation, ou rendus flous par de brusques changements de mise au point. Une telle primauté accordée au visage n'est pas indépendante des expériences du cinéaste sur la représentation de l'Histoire et la mise en cause du pouvoir des médias qui l'ont rendu célèbre. Le visage est, bien sûr, le lieu où, de 
manière fantasmatique, la parole se donne à voir. Qui parle? Qui est autorisé à prendre la parole? sont alors des questions pressantes que le spectateur est invité à se poser devant ces films. Entre les deux figures du témoin et de l'expert, le réalisateur opère des déplacements qui invitent à s'interroger sur chacune de ces positions, comme sur le rôle et le statut de la voix over qu'on entend sans visualiser le corps qui est à la source de son émission. L'efficacité du cinéma de Watkins repose sur des questions simples maintenues avec opiniâtreté et rigueur - celle qui porte sur le corps parlant retiendra mon attention.

\section{Du visage}

La valorisation du visage se traduit de manière assez nette par la restriction des plans larges, en particulier ceux qui pourraient être associés à des motifs paysagers. Un passage est révélateur à ce propos dans Edvard Munch (1973): le peintre, alors jeune homme, est reçu chez Madame Heiberg, femme libre qui exerce un puissant attrait sur lui. Mangeant sans appétit, pour trouver un motif insignifiant de discussion et faire bonne figure, il parle soudain du "merveilleux paysage" (hors de la maison) avant de retomber dans le silence, ce qui provoque l'hilarité de la jeune femme. Nous ne voyons pas le paysage au moment de cet échange, et nous l'entrevoyons à peine dans les quelques plans suivants qui montrent les deux personnages se promenant dans des sous-bois, puis au bord du fjord de Kristiana. Si ces plans sont bien situés en extérieur, le réalisateur a privilégié le gros plan, voire le très gros plan quand Munch ose embrasser Madame Heiberg dans le cou - inversant alors le motif de la femme-vampire présent dans l'œuvre du peintre. Et quand le cadre est moins serré sur les comédiens, la focale utilisée limite la visibilité de la profondeur de champ. La simple mention du paysage prête donc bien au fou rire tant elle paraît incongrue. Le fragment mentionné n'a pas une simple valeur ponctuelle, car on retrouve une telle mise à l'écart

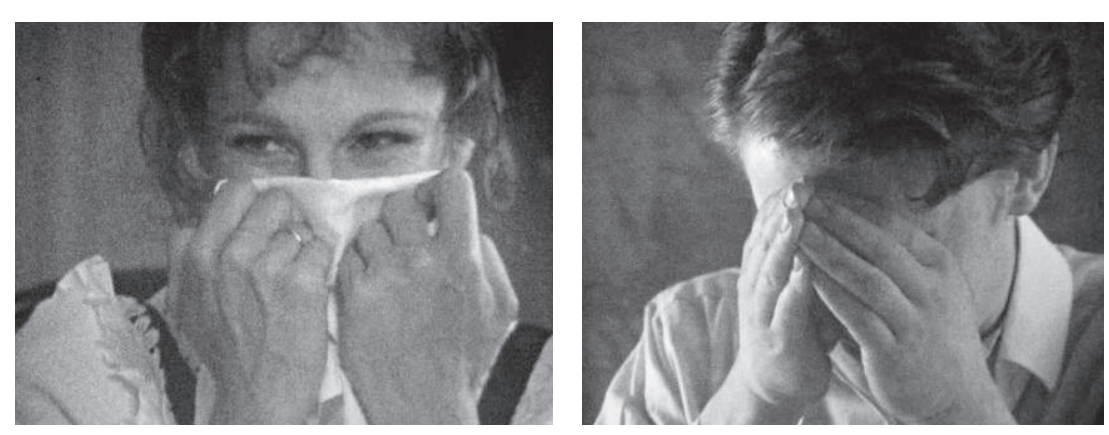



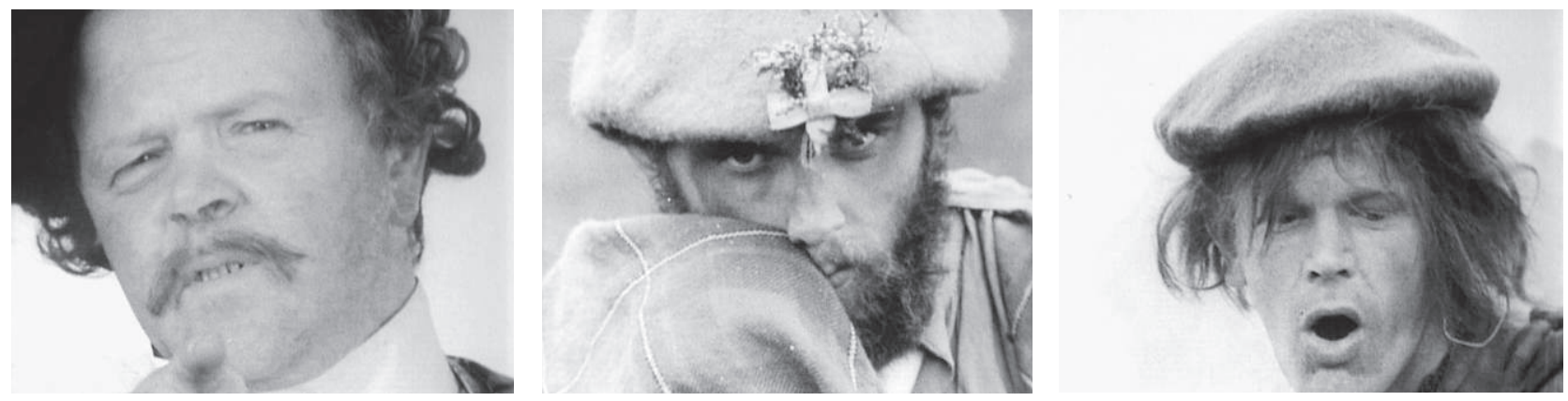

Culloden (1964)
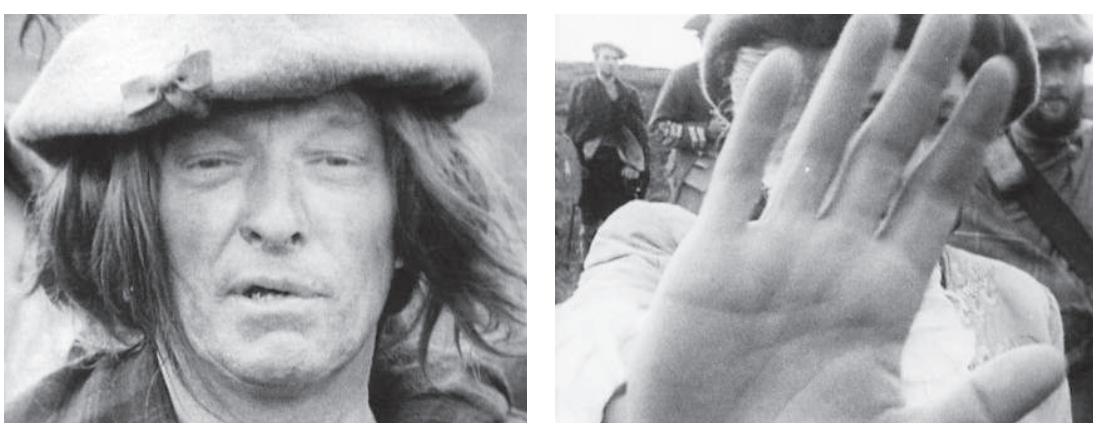

3 A titre de contraste entre la pratique effective du peintre et ce qu'en retient le cinéaste, on peut renvoyer au tableau de Munch Jour de printemps sur l'avenue Karl Johan (1890), qui use d'une perspective irréaliste mais très marquée.

du paysage de façon généralisée, par exemple dans ces plans récurrents montrant la rue Karl Johan, promenade obligée pour la bonne société de la ville, jamais montrée dans sa perspective, mais apparaissant comme une accumulation de corps en mouvement, filmés en plans serrés.

Watkins s'abstient donc de traiter la question du paysage dans un film de 3 heures 30 évoquant la vie d'un peintre qui en a tout de même représenté un certain nombre ${ }^{3}$. Ou alors il l'utilise de manière homéopathique, sous un mode qui voisine avec le cliché. Ainsi est-ce le cas quand au bord d'un fjord des arrière-plans à la tonalité orangée et violette s'apparentent à une toile de fond, évoquant la peinture de Munch, et ne se présentent pas comme vue paysagère.

De façon analogue, dans Culloden (1964), la présentation initiale du champ de bataille semble neutraliser la présence du lieu, et empêcher toute possibilité d'un plan-paysage: après un plan initial sur des soldats en armes avançant vers la caméra, un panoramique hâtif, un peu heurté, balaie la lande au moment où la voix over précise l'emplacement du conflit décisif entre les anglais loyalistes et les partisans de Charles Stuart, prétendant catholique au trône. L'image en noir et blanc est peu contrastée, amplifiant l'aspect morne de la lande et confirmant son usage fonctionnel dans le film. Par contraste, c'est une succession de visages 
qui occupe cette ouverture: nous voyons d'abord quatre dirigeants de l'armée jacobite, singularisée par un zoom avant sur leur visage, alors que la voix over, sarcastique, les présente en tournant en dérision leur incompétence. Avant le générique, suivent quatre autres gros plans, où le seul stratège talentueux du camp jacobite, puis un soldat montré deux fois, et «Bonnie Prince Charlie» s'adressent à tour de rôle à la caméra. Pour Watkins, il ne s'agit pas d'être sur le lieu même de l'événement évoqué, à l'opposé de la pratique de Straub et Huillet pour prendre un seul exemple. Canterbury vaut Budapest, comme n'importe quel lieu peut être utilisé comme décor destiné à l'apparition d'une série de faces humaines. Dans Culloden, l'utilisation abondante de la fumée des armes à feu renforce l'idée que l'espace diégétique est devenu presque abstrait: ce n'est qu'un milieu pour les corps enregistrés. Le film repose ainsi sur un enchaînement de plans-visages. Que les visages soient muets ou bavards, on ne peut manquer d'éprouver une sensation d'étouffement en raison de la proximité avec laquelle ils sont rendus. L'oppression se double toutefois d'une sorte d'énigme composée par ces singularités ainsi juxtaposées les unes aux autres.

Par antithèse, The War Game (1965) s'ouvre sur un motard de dos dont on suit la course jusqu'à la porte d'un bâtiment officiel, puis la montée d'escalier jusqu'à son entrée dans la salle où se tient le comité en charge de l'évacuation des civils depuis la déclaration de l'état d'urgence - comme l'explique de manière précise la voix over. Puis, de manière brutale, on passe à un plan sur le directeur du comité, filmé de face, énumérant les différentes catégories de population prioritaires. La rupture est définitive puisque désormais vont se succéder des séries de visages, présentées selon des modalités d'énonciation différentes (déclaration, entretien, témoignages, ou portraits muets) qui structurent le reste du film.

Une telle insistance sur le visage évoque, sur un matériau qui en semble loin (en particulier dans The War Game ou Punishment Park, 1970), l'époque du muet et, plus encore, la valorisation du gros plan par certains théoriciens des années 1920. On pourrait imaginer un dispositif à la Watkins où un acteur se projetant dans le rôle de Béla Balász visionnerait et commenterait certains de ses films avec exaltation. En effet, on retrouve chez le cinéaste l'idée, formulée par Balász, que le visage en gros plan doit être isolé de son environnement pour être montré dans ses moindres détails, en rupture avec le reste du film, c'est-à-dire hors du strict déroulement narratif. Cette élection du visage permettrait-elle au drame de se refléter dans les traits du visage dont, par synecdoque, celui-ci deviendrait "la totalité»? Sans nul doute, cette paraphrase de $L^{\prime}$ 'homme visible $\mathbf{4}$ pourrait s'appliquer aussi bien à Forgotten Faces qu'à The
4 Béla Balázs, L'homme visible et l'esprit du cinéma, Paris, Circé, 2010 [Der Sichtbare Mensch, oder die Kultur des Films, Vienne/Leipzig, Deutsch-Österreichischer Verlag, 1924]. 
5 Béla Balázs, L'esprit du cinéma, Paris, Payot, coll. "petite bibliothèque Payot", 2011, p. 192 [Der Geist des Films, Halle, Wilhelm Knapp, 1930].

6 Peter Watkins, "Auto-interview", dans le livret du DVD Edvard Munch, Doriane Film, 2005.
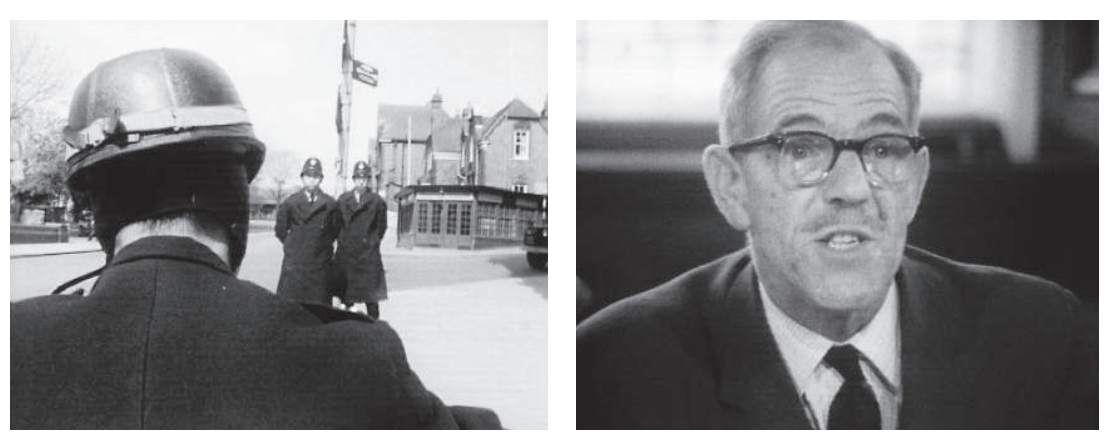

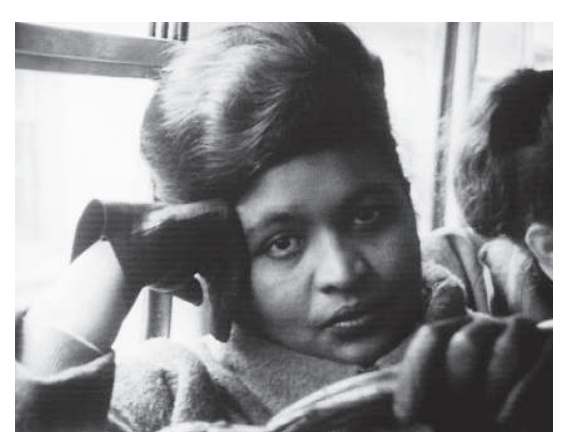

War Game, à Culloden qu'à Edvard Munch, le mot drame n'étant pas de trop tant, chez Watkins, la valorisation des visages permet de concentrer l'essentiel du propos. L'auteur de L'esprit du cinéma écrivait également, en commentant le jeu de l'acteur Sessue Hayakawa, que «nous voyons clairement sur ce visage qu'il n'y a rien à voir sur ce visage. Quelque chose existe qui n'est pas localisable. Une expression distinctement invisible.» $\mathbf{5}$ Cette formule, paradoxale en apparence, décrit assez bien, si on en détourne en partie la signification originale, la pratique de Watkins : en effet, interprètes ou figurants imposent leur présence, leur visage étant le détail privilégié de l'expression de l'individu. Dans le même temps, il s'agit d'un visage qui joue, qui tient un rôle, d'où cette tension productive où l'effet de présence est sans cesse renvoyé à l'artifice d'une représentation.

Parlant du casting d'Edvard Munch, Watkins insistait sur l'importance dévolue au visage pour justifier son choix des comédiens ou figurants :

«Nous procédions notamment par la compilation de livres, représentations et biographies sur toutes les personnalités et figures importantes de la vie de Munch. Nous les apportions aux réunions de casting, où nous étudiions attentivement le visage de chaque personne que nous rencontrions pour le comparer à notre collection de portraits. La plupart des personnes dans le film présentent une ressemblance physique extraordinaire avec les gens qu'ils jouent, ce qui est très important, la ressemblance physiologique s'accompagnant souvent d'une ressemblance psychologique. » $\mathbf{6}$

Le réalisateur britannique, là encore, semblerait en accord avec le théoricien hongrois qui posait comme conséquence de la «microphysionomie» offerte par le cinéma la possibilité d'une «micropsychologie». On constate toutefois que le réalisateur part de représentations, d'images (de même qu'il s'était inspiré très précisément de photographies de 
presse pour Forgotten Faces), que les interprètes doivent incarner: ce n'est donc pas tant une plongée dans les profondeurs d'un sujet, qu'un jeu de surface - en tant qu'elle est aussi expression de l'individu. Dans bien des cas, l'interprète doit alors prêter une voix à celui qu'il incarne, généralement un disparu. Watkins explique également que dès ses premiers essais il voulait "substituer au processus artificiel d'Hollywood les visages et émotions de personnes authentiques. C'était notamment l'ambition d'un film comme The Forgotten Faces, où j'ai travaillé avec des gens ordinaires pour recréer les événements du soulèvement de 1956 en Hongrie comme s'ils se déroulaient devant les caméras des actualités » $\mathbf{7}$. Cette précision indique bien le dialogue qui s'opère entre recherche d'authenticité et travail de représentation.

\section{De la prosopopée}

L'élection du visage repose sur la frontalité du cadrage et l'adresse fréquente à la caméra qui indiquent l'importance de la prise de parole. La plupart de ces visages sont associés à un nom, qui leur est attribué par la voix over (que les personnes soient réelles ou fictives) - ce principe utilisé dès Forgotten Faces est repris dans les films ultérieurs. Le témoignage est donc fictionnalisé, il est bien du ressort d'un comédien, même si, dans la même opération, il peut rendre perceptible quelque chose d'indistinct appartenant à celui qui en a la charge. Une comparaison serait sans doute fructueuse avec Godard et sa pratique des interviews fictives dans les films contemporains de Culloden ou The War Game, par exemple Masculin-féminin (1966), Deux ou trois choses que je sais d'elle (1967) ou La Chinoise (1967).

Ces corps, ces visages apparaissent ainsi sur le mode du dédoublement. Celui-ci ne tient pas seulement à l'opposition entre l'image et le son (entre visage et discours) mais à la tension entre l'effet-de-présence d'une singularité, dont le visage est la présence la plus immédiate et la plus irréductible, et une parole qui renvoie à l'art du comédien. On le voit dès le début de Culloden, mentionné plus avant, comme dans The War Game, avec les témoignages reconstitués, qui s'affichent comme tels - avec parfois une pointe d'humour comme lors de l'évocation du coût d'un abri antiatomique à domicile. Certes, les déclarations du cinéaste ou des témoignages extérieurs nous apprennent que dans Culloden les jacobites sont interprétés par des Ecossais dont certains sont les descendants des familles persécutées au XVIII siècle, mais aucun critère objectif ne permet au regard d'opérer une telle discrimination devant le film. Dans Edvard Munch, la stratégie consiste parfois à laisser affleurer dans certaines prises de position reconstituées une actualité
7 Peter Watkins, Media crisis, Paris, Homnisphères, 2004, p. 13 [2003, cf. http://pwatkins. mnsi.net/part1_home.htm]. 
8 Voir, par exemple, Theodor W. Adorno, Minima Moralia, Paris, Payot, coll. "petite bibliothèque Payot", 2003, § 97 "la monade", pp. 200-203 [Minima Moralia. Reflexionen aus dem beschädigten Leben, Frankfurt am Main, Suhrkamp Verlag, 1951].

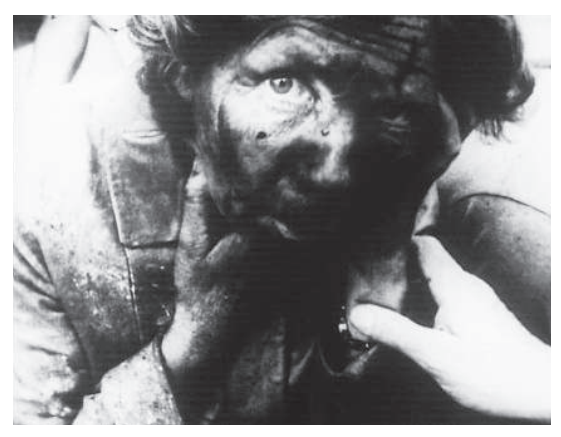

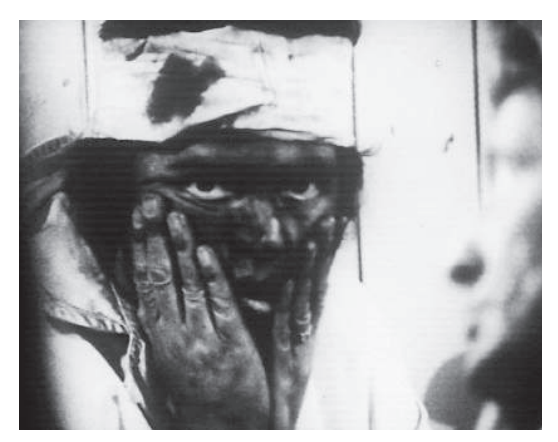

évidente. C'est le cas lorsque les maîtresses du peintre parlent de leur relation aux hommes, à l'amour, au mariage, en faisant explicitement écho aux revendications contemporaines à la réalisation du film. Mais l'effet produit reste le même: il y a une réversibilité entre le témoignage d'un individu et la représentation d'un personnage.

Les plans pris dans la rue dans The War Game offrent une variété de visages tout à fait saisissante, mais celle-ci s'inscrit dans la logique dialectique du montage visant à opposer la méconnaissance du danger atomique, dont témoignent la majorité des propos, et l'horreur d'une attaque nucléaire illustrée par le film, ou bien à renvoyer les préjugés (la nécessité d'une vengeance) à leurs conséquences (un engrenage meurtrier). Suivant cette logique, les figurants interrogés apparaissent moins comme des singularités que des porte-parole de lieux communs - contrairement à ce qu'on trouve par exemple dans Chronique d'un été (Jean Rouch, Edgar Morin, 1960) ou Le joli mai (Chris Marker, 1963), juste antérieurs. Leurs propos sont au même niveau que les prises de parole mises en scène, désignées de manière explicite par l'insertion d'intertitres signalant la véracité des paroles employées par les acteurs jouant un faux ecclésiastique ou un spécialiste en stratégie militaire de manière caricaturale. Faut-il comprendre que l'entretien ne fait que reproduire l'idéologie dominante? Ou, en suivant Adorno, constater la ruine du sujet désormais entièrement aliéné ? 8 Si l'on s'attarde sur ce film, les visages les plus impressionnants, les plus présents en mémoire sont ceux qui mettent en évidence une expressivité marquée : la femme suffocant au visage noirci, cet enfant se cachant les yeux avec ses mains, cet homme n'arrivant pas à boire sa soupe, etc. Leur pouvoir de suggestion repose sur le maquillage, le mime appuyé de la souffrance, de l'étouffement, de l'état de choc. Leur visage devient un masque de convention, mais ni l'émotion ni la démonstration ne sont pour autant congédiées et désamorcées. Pour cette raison, il est tentant de rappeler le

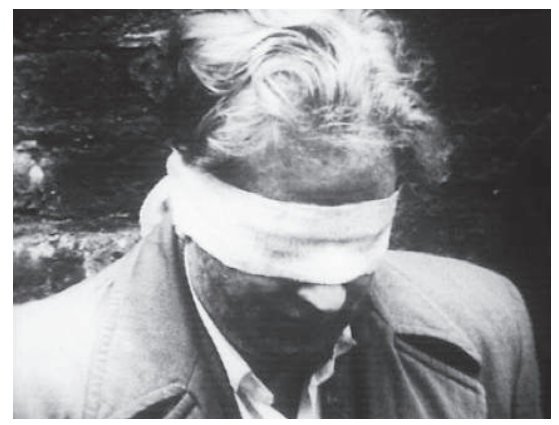


terme grec prosôpon, qui dit à la fois le visage (de l'individu) et le masque (de l'acteur, du personnage). C'est à partir de ce terme, que l'on parle en rhétorique de prosopopée - que l'on pourrait traduire littéralement par «faire visage» (prosôpon + poïen). Voici la définition qu'en propose Fontanier dans Les figures du discours:

"La Prosopopée, qu'il ne faut confondre ni avec la Personnification, ni avec l'Apostrophe, ni avec le Dialogisme, qui l'accompagnent presque toujours, consiste à mettre en quelque sorte en scène, les absents, les morts, les êtres surnaturels, ou même les êtres inanimés ; à les faire agir, parler, répondre, ainsi qu'on l'entend; ou tout au moins à les prendre pour confidents, pour témoins, pour garants, pour accusateurs, pour vengeurs, pour juges, etc. ; et cela, ou par feinte, ou sérieusement, suivant qu'on est ou qu'on n'est pas le maître de son imagination." $\mathbf{9}$

Les absents sont bien convoqués par Watkins qui donne ainsi à éprouver la présence du masque et l'individu qui la supporte. Malgré la diversité de leurs interventions, les victimes de la bataille de Culloden et de la "pacification" qui l'a suivie, comme les irradiés atomiques, ou la sœur morte de Munch s'expriment bien comme les porte-voix de morts passés et encore en sursis.

\section{La voix over (comme risque)}

Dans les films de Watkins, les visages sont tournés vers la caméra; on pourrait croire qu'ils nous regardent. Si l'interpellation semble fonctionner il ne faut pas négliger que cet usage n'en est pas moins présent dans l'histoire du cinéma, pour ne rien dire de la rhétorique télévisuelle. Ainsi, la lecture que François Niney fait de ce procédé dans Edvard Munch peut-elle être nuancée ${ }^{\mathbf{1 0}}$ : ce n'est pas toujours ou pas seulement le spectateur qui est ainsi interpellé par les regards caméra (ou vers la caméra), transformant le hors-champ en hors-cadre. Le regard à la caméra est, en effet, si systématique qu'il revêt une valeur dynamique: autant qu'une rupture de l'illusion représentative, il s'agit d'un moyen permettant de rapprocher des événements distants dans le temps. Par exemple, le regard de Munch adulte est relié au regard frontal du même personnage enfant ; ou encore, par un semblable parti pris frontal, le regard permet de passer d'une scène de cabaret à un plan sur la promenade Karl Johan, ou du travail en atelier à une scène d'enfance du peintre. En mêlant les temporalités et en faisant revenir les mêmes figures obsédantes de l'enfance, l'organisation du montage donne également l'impression que Munch agit sous le regard de quelques images traumatiques (la sœur agonisant et crachant du sang, lui-même souffrant de tuberculose). Sans doute
9 Pierre Fontanier, Les figures du discours, Paris, Flammarion, 1977, p. 404 [ce recueil rassemble le Manuel classique pour l'étude des tropes, Paris, Belin-Leprieur, 1821 et Des Figures du discours autres que les tropes, Paris, Maire-Nyon, 1827].

10 François Niney, "A travers la toile du temps: Edvard Munch, la danse de la vie", Théorème, $n^{\circ}$ 14, Presses de la Sorbonne Nouvelle, 2011, pp. 69-75. 

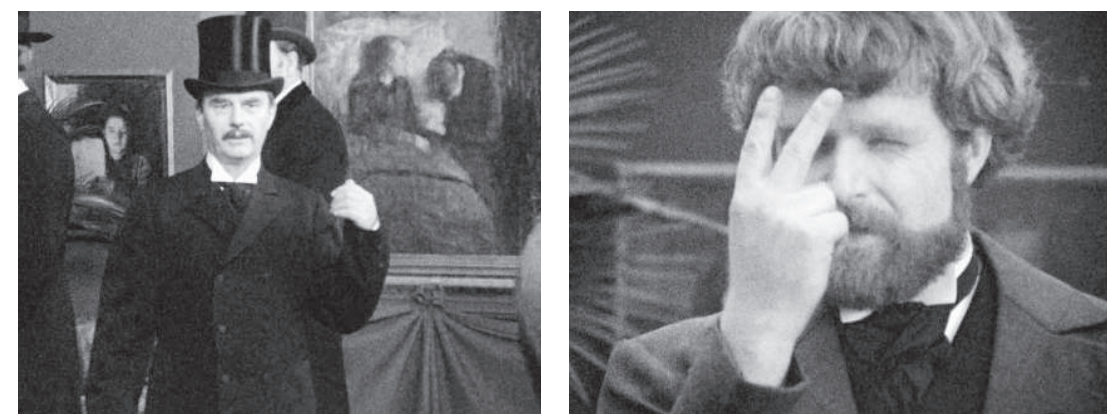

s'agit-il pour Watkins de faire sentir le dispositif pictural en insistant sur un regard renvoyant au portrait, mais au-delà il s'agit bien de mettre en scène les absents ou les morts à l'origine de la créativité du peintre: ce n'est pas le spectateur qui est ainsi visé mais un ailleurs, plus angoissant et moins localisable. Ne pourrait-on d'ailleurs émettre l'hypothèse que la voix over elle-même subirait cette apostrophe fréquente des personnages? Plutôt que de se cantonner au face-à-face supposé entre spectateur et personnage, on n'oublierait pas alors l'instance protégée par son invisibilité. De la sorte, une distance ironique apparaît de manière plus évidente entre le peintre et la voix officielle qui énonce les aspects majeurs de sa biographie.

Présente notamment dans The War Game, Culloden, Edvard Munch, la voix over tend en apparence à neutraliser la présence du corps. Son rôle est déterminant dans la mise en question de la forme documentaire et l'écriture de l'histoire. Et on peut aussi penser qu'elle prend en charge ce hors-champ diégétique neutralisé, en partie, par l'inflation des plans de visage. Dans Munch, elle se fait de plus en plus présente au cours du film, à mesure que la lecture des écrits intimes du peintre se raréfie. Cette voix ne fournit pas seulement informations et précisions, elle vient parfois couvrir bruits et propos des personnages, par exemple dans certains fragments de la bohème berlinoise au cabaret du Cochon noir. La voix over dans Munch, comme celle de The War Game, ou de Culloden, ou auparavant de Forgotten Faces est une voix masculine, au débit parfois rapide, mais avec une articulation parfaite des syllabes. Haut perchée dans Edvard Munch, elle n'en conserve pas moins l'apparence d'une voix de commentaire traditionnelle, assurée, sans hésitation, conservant un ton sentencieux même lorsque le texte lu est factuel - voix de l'autorité et du savoir qui feint d'être désincarnée, parfait fantasme d'une voix neutre que Barthes associait à une sensation de «grande terreur» $\mathbf{1 1}$. Toutefois, il ne faut pas oublier la dimension ironique, voire parodique
11 " [...] il n'y a pas de voix neutre - et si parfois ce neutre, ce blanc de la voix advient, c'est pour nous une grande terreur, comme si nous découvrions avec effroi un monde figé, où le désir serait mort." (Roland Barthes, "La musique, la voix, la langue", L'obvie et l'obtus, Essais critiques III, Paris, Seuil, 1982, p. 247). 
de ce type d'intervention. Comme le rappelle De Baecque, à la suite d'autres commentateurs, dans The War Game la voix over est celle "d'un présentateur de la BBC bien connu, Michael Aspel, parodiant lui-même un autre célèbre présentateur britannique, officiant pendant la Seconde Guerre mondiale, Dick Graham»12. Dans un ordre comparable, comment entendre la litanie de dates égrenées dans Edvard Munch par cette voix over "encyclopédique", selon la formule de Niney, qui opère une juxtaposition de faits anecdotiques (la mort de Tchaïkovski et la naissance de Göring mentionnées l'une après l'autre, pour ne prendre qu'un exemple parmi des dizaines d'autres)? N'est-ce pas la vanité de l'omniscience qui est ainsi tournée en dérision de manière récurrente en pointant la cécité et la partialité du narrateur? De manière différente, la voix over de Culloden affiche en permanence son parti pris, jugeant sans nuance les inconséquences du clan jacobite, puis condamnant par des formules sèches la "pacification» de l'Ecosse par les troupes anglaises. L'idée d'objectivité est ainsi congédiée pour signaler qu'un commentaire est forcément situé ou engagé.

Comme le précisait Serge Daney, pour que la voix over perde son pouvoir illimité, il faut qu'elle "risque quelque chose et le risque en tant que voix»13. Outre les procédés mentionnés, il faut considérer les relations tissées entre les différentes prises de parole afin de bien mesurer le rôle stratégique accordée à la parole par Watkins. Prenons, dans Edvard Munch, le passage consacré à la première exposition du peintre. La voix du narrateur fait une brève présentation didactique sur la situation de la peinture à l'époque où Munch achève son premier grand tableau, L'enfant malade (1886). On a l'équivalent d'une conférence avec images et commentaire savant, relativement banal (et d'ailleurs assez contestable dans l'équivalence proposée entre les différents peintres), comme pouvait le faire à l'époque du peintre un conférencier-lanterniste. Suivent des plans sur l'exposition : après un plan sur le tableau, un acteur jouant un visiteur donne son opinion (négative), face à la caméra. Ces sentences sont redoublées par le narrateur lui-même qui cite, au style direct, des propos tout aussi critiques d'amis ou de proches du peintre. Par contraste, s'insèrent dans cet ensemble des interventions de Hans Jaeger, l'ami et mentor libre-penseur, qui fait des déclarations enflammées sur la liberté d'expression depuis la cellule où il est incarcéré. Puis, élément supplémentaire, vient s'ajouter l'intervention d'un historien d'art introduit par la voix over, filmé de manière frontale, en position d'adresse à la caméra. Cet expert, dont le propos est lui très laudateur, compose une anticipation du goût contemporain, puis il intervient à nouveau sur des plans, déjà vus auparavant: l'agonie de la sœur tuberculeuse crachant du
12 Antoine De Baecque, "Peter Watkins en direct de l'histoire", op. cit., p. 217.

13 Serge Daney, "L'Orgue et l'aspirateur" [Cahiers du cinéma, n 279-280, aoûtseptembre 1977], La rampe, Paris, Petite bibliothèque des Cahiers du cinéma, 1996, p. 172 [La rampe, Paris, Gallimard, 1983]. 
sang, le bain de ses deux sœurs. Il faut de "nouvelles paires de lunettes» dit-il, alors que le montage offre une explication qui fait de ces deux événements (psychiques) la source du tableau, et secondairement de la névrose de l'artiste. Introduit par la voix over, on peut dire que l'historien d'art en reprend le ton et la fonction dans l'espace fictionnel, devenant à la fois guide et pédagogue. Mais ce délégué de la voix over est aussi celui qui en permet la dispersion. Les opinions des spectateurs, le jugement du spécialiste, la voix du narrateur, les propres réflexions de Munch se succèdent de manière rapide de façon à produire une véritable incertitude sur la relation entre les images et les propos. Il ne s'agit pas d'une autonomie mais plutôt d'un flottement orchestré entre les images et les discours - quelles sont ces voix qui parlent derrière, ou par-dessus, ces visages aux allures de masques?

Le dernier exemple mentionné permet d'insister sur l'impression de polyphonie, fréquente dans l'œuvre de Watkins, et qui forme un de ses horizons essentiels. Les paroles prolifèrent, quel que soit leur mode d'énonciation, tandis que se succèdent des images de visages, telle pourrait être la formule condensant les quelques films évoqués. D’où le choix d'utiliser cette figure de la prosopopée: il s'agit bien de donner corps à une voix, que celle-ci rende justice aux absents ou laisse entendre une parole d'accusation portant sur l'état des sociétés humaines. Fontanier précisait que les véritables prosopopées étaient le «fruit d'une imagination un peu libre». L'aptitude à la libre imagination permet sans nul doute à cette œuvre hantée par la mort et la destruction de dessiner un espace collectif où ces puissances se trouvent, au moins pour un temps, maintenues à distance. 